\author{
Nickolai Kostin \\ Tatyana Mishchenko \\ Andrey Petrov \\ Dnepropetrovsk National University \\ of Railway Transport named after \\ Academician V. Lazaryan (Ukraine) \\ Oksana Reutskova \\ Dnepropetrovsk National University (Ukraine)
}

\title{
Non-productive losses of electric power in the networks of traction power supply of main railways
}

\begin{abstract}
A new method of correlation functions, determinations of power indexes and nonproductive (additional) losses of electric power in the traction electric systems of direct-current have been suggested. Non-productive losses have been calculated for a number of feeder areas. It has been shown that these losses are $6 . .15 \%$ of electric power consumed by electric train.
\end{abstract}

\section{Introduction}

The preset work is continuation and development of the researches [1] of efficiency of electric energy processes in the system of electric traction of direct current of main railways.

As it is generally known, at the power transmission from traction substation to electric train, the losses of active electric power have place in the wires of traction network. These losses are called technological and can be productive (basic) and non-productive (additional).

Productive losses are conditioned by a transmission of only active power and they take place when the system of electric traction works in a sine wave, symmetric and stable mode by the nature of consumption. These losses are necessary and inevitable for electric power transmission. Therefore, they must be reduced to the certain optimum level.

Non-productive losses are conditioned by a transfer in wires of traction network of inactive power, that they are related to a poor quality of electric power. Therefore, the task of estimate of these losses (as the component of general losses) and their reduction practically to the zero is the major task to increase efficiency of traction electric supple. Presently, as it is generally known, there are not either devices or methods of the direct measurement of not only nonproductive but also in general total technological losses. Therefore, in this work the method of the indirect (indirect) measurement of the investigated losses is applied on the basis of experiment, in the real conditions of exploitation, registered time dependences of feeder voltages and currents.
2. Method of correlation functions of nonproductive losses determination

According to [1], non-productive losses of electric power $\Delta W_{\text {ad }}$ for the time interval $\tau$ are proportional to the square of reactive power after Fryze $Q_{F}^{2}$ :

$$
\Delta W_{\mathrm{ad}}=R_{\Sigma} \cdot \frac{Q_{F}^{2}}{U^{2}} \cdot \tau=R_{\Sigma} \cdot \frac{P^{2} \cdot \operatorname{tg}^{2} \varphi}{U^{2}} \cdot \tau,
$$

where $R_{\Sigma}$ - total (equivalent) resistance of a traction network and substation;

$U$ - effective value of feeder (substation) voltage;

$P$ - transferrable active power;

$\operatorname{tg} \varphi$ - coefficient of reactive power.

A similar formula is derived for basic, productive losses $\Delta W_{\mathrm{b}}$.

As it follows from (1), the task of determination of $\Delta W_{\text {ad }}$ is closely related to the task of determination of inactive power, and the last one is not simple as feeder tensions $U(t)$ and current $I(t)$ are random processes (fig.1)

Formula [1] offers two methods to find powers $Q_{F}$, $P$ : the method of discrete transformation of Fourier and the «discrete electrical engineering» method. This work offers a new method of correlation functions, which is based on the known in the theory stationary random processes, concepts of auto- and mutual correlation functions $[2,3]$. 
a)

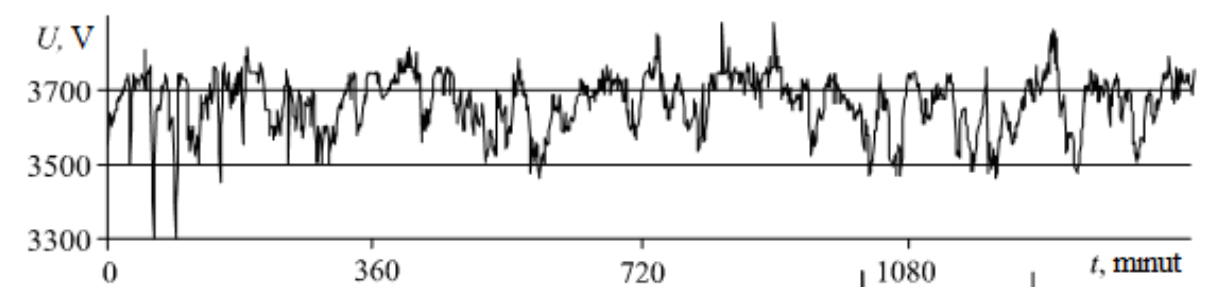

b)

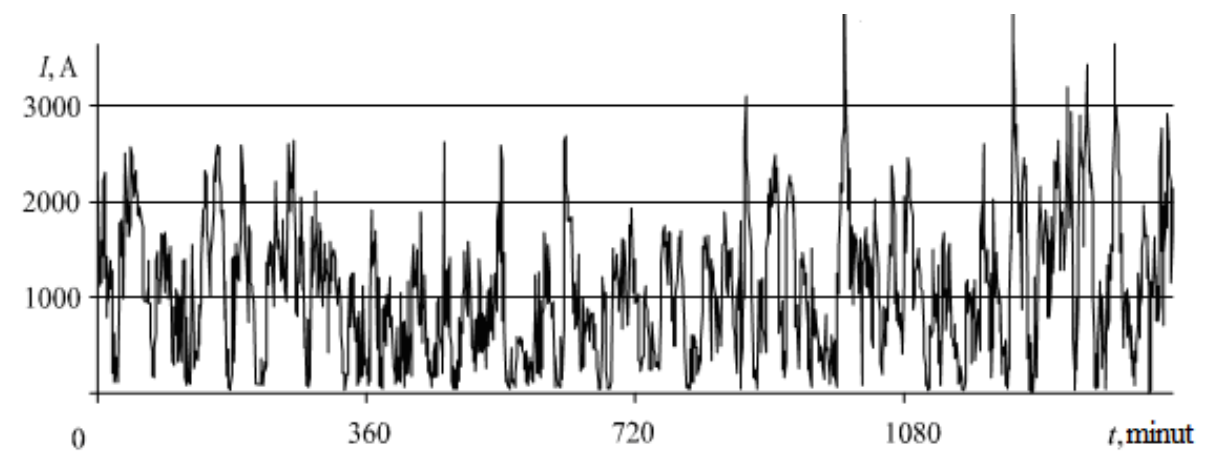

Fig.1 - Time dependences of rectified feeder voltage (a) and current (b)

According to this theory, autocorrelation function $\hat{E}_{U}(\tau)$ of voltage $u(t)$, as a stationary ergodic random process, it is possible to write down as the expectation of scalar work of the centring random function $\stackrel{\circ}{U}(t)$ and it's shifted for the interval of correlation $\tau$ of the copy $\stackrel{\circ}{U}(t+\tau)$ :

$$
\begin{aligned}
K_{U}(\tau) & =M[\stackrel{\circ}{U}(t) \cdot \stackrel{\circ}{U}(t+\tau)]= \\
& =M\left\{\left[U(t)-m_{U}\right] \cdot\left[U(t+\tau)-m_{U}\right]\right\},
\end{aligned}
$$

where $m_{U}$ - expectation of stationary random function of voltage $U(t)$ (constant).

Similarly, the autocorrelation function $\hat{E}_{2}(\tau)$ of random function of current $\hat{E}_{2}(\tau)$ is:

$$
\begin{aligned}
K_{I}(\tau) & =M[\stackrel{\circ}{I}(t) \cdot \stackrel{\circ}{I}(t+\tau)]= \\
& =M\left\{\left[I(t)-m_{I}\right] \cdot\left[I(t+\tau)-m_{I}\right]\right\},
\end{aligned}
$$

where $m_{I}$ - expectation of stationary random function of voltage $I(t)$ (constant).

For stationary random processes in very wide terms [2], the unbiased estimate of expectation $\grave{I}[X(t)]$ of any stationary function $X(t)$ is proved to be its mean value by time $\bar{x}$ of realization by the duration $T$ of the function $X(t)$, that's

$$
M[X(t)]=m_{x}=\bar{x}=\frac{1}{T} \int_{0}^{T} x(t) d t .
$$

Then, in accordance with (4), expressions (2) and (3) for correlation functions can be written as:

$$
\begin{aligned}
& K_{U}(\tau)=\frac{1}{T} \int_{0}^{T}\left[U(t)-m_{U}\right] \cdot\left[U(t+\tau)-m_{U}\right] d t= \\
& \frac{1}{T} \int_{T}^{1} U(t) \cdot U(t+\tau) d t- \\
& -\frac{1}{T} \int_{0}^{T} U(t) \cdot m_{U} d t-\frac{1}{T} \int_{0}^{T} U(t+\tau) \cdot m_{U} d t+m_{U}^{2},
\end{aligned}
$$

Similarly,

$$
\begin{gathered}
K_{I}(\tau)=\frac{1}{T} \int_{T}^{1} I(t) \cdot I(t+\tau) d t- \\
-\frac{1}{T} \int_{0}^{T} I(t) \cdot m_{I} d t-\frac{1}{T} \int_{0}^{T} I(t+\tau) \cdot m_{I} d t+m_{I}^{2} .
\end{gathered}
$$

The autocorrelation function determines the law, characteristic of only one process $\left(u(t)\right.$ or $\left.{ }^{3}(t)\right)$ and is used to find effective values of sizes. Indeed, at $\tau=0$ expressions (5) and (6) are:

$$
\begin{gathered}
K_{U}(\tau=0)=\frac{1}{T} \int_{0}^{T}\left[U^{2}(t)-m_{U}^{2}\right]=U^{2}-m_{U}^{2}, \\
K_{I}(\tau=0)=\frac{1}{T} \int_{0}^{T}\left[I^{2}(t)-m_{I}^{2}\right]=I^{2}-m_{I}^{2} .
\end{gathered}
$$

where $U$ and $I$ - effective values of voltage and current, found with temporary realizations with the duration $\dot{O}$.

Taking into account (7) and (8), total power is determined by autocorrelation function as

$$
S=U \cdot I=\sqrt{\begin{array}{l}
\left.K_{U}(\tau=0)+m_{U}^{2}\right] \times \\
\times\left[K_{I}(\tau=0)+m_{I}^{2}\right]
\end{array}}
$$


If in the expression (2) in order to replace the shifted copy of voltage $\stackrel{\circ}{U}(t+\tau)$ with the shifted copy of current $I(t+\tau)$, we will get the mutual correlation function of voltage with the current:

$$
\begin{gathered}
K_{U I}(\tau)=M[\stackrel{\circ}{U}(t) \cdot \stackrel{\circ}{ }(t+\tau)]= \\
=M\left[\left(U(t)-m_{U}\right) \cdot\left(I(t+\tau)-m_{I}\right)\right]= \\
=\frac{1}{T} \int_{0}^{T}\left\{\left[U(t)-m_{U}\right] \cdot\left[I(t+\tau)-m_{I}\right]\right\} d t= \\
=\frac{1}{T} \int_{0}^{T} U(t) \cdot I(t+\tau) d t- \\
-\frac{1}{T} \int_{0}^{T} U(t) \cdot m_{I} d t-\frac{1}{T} \int_{0}^{T} m_{U} \cdot I(t+\tau) d t+ \\
+\frac{1}{T} \int_{0}^{T} m_{U} \cdot m_{I} d t
\end{gathered}
$$

Similarly, if in the expression (2) to replace the shifted copy of current $I(t+\tau)$ with the shifted copy of voltage $\stackrel{\circ}{U}(t+\tau)$, we will get the mutual correlation function of current with the voltage:

$$
K_{I U}(\tau)=M[\stackrel{\circ}{U}(t+\tau) \cdot \stackrel{\circ}{I}(t)] .
$$

At a temporary shift change equal to the zero, formulas (10) and (11) determine energy of voltage and current interaction, expressed by mutual correlation functions:

$$
\begin{aligned}
& K_{U I}(\tau=0)=\frac{1}{T} \int_{0}^{T} U(t) \cdot I(t) d t-m_{U} \cdot m_{I}, \\
& K_{I U}(\tau=0)=\frac{1}{T} \int_{0}^{T} I(t) \cdot U(t) d t-m_{I} \cdot m_{U} .
\end{aligned}
$$

At the same time it is also known that according to the theoretical electrical engineering, active-power $Ð$ is determined as arithmetical mean of instantaneous power $p(t)=U(t) \cdot I(t)$ for the interval $[0, T]$ :

$$
P=\frac{1}{T} \int_{0}^{T} p(t) d t=\frac{1}{T} \int_{0}^{T} U(t) \cdot I(t) d t,
$$

Comparing expressions (12) and (13) with the formula (14) we come to the conclusion that active-power $D$ is determined by the mutual correlation function of voltage with a current $K_{U I}(\tau)$ or current with voltage $K_{I U}(\tau)$ at a temporary shift $\tau=0$ as

$$
P=K_{U I}(\tau=0)+m_{U} \cdot m_{I} .
$$

$$
\begin{aligned}
Q_{F} & =\sqrt{\begin{array}{l}
\left(K_{U}(\tau=0)+m_{U}^{2}\right) \times \\
\times\left(K_{I}(\tau=0)+m_{I}^{2}\right)- \\
-\left[K_{U I}(\tau=0)+m_{U} \cdot m_{I}\right]^{2}
\end{array}}= \\
= & \sqrt{\begin{array}{l}
\left(D_{U}+m_{U}^{2}\right) \cdot\left(D_{I}+m_{I}^{2}\right)- \\
-\left[K_{U I}(\tau=0)+m_{U} \cdot m_{I}\right]^{2}
\end{array}}
\end{aligned}
$$

\section{Results of numeral calculations and their ana- lysis}

With the use of the above-mentioned correlations productive and non-productive losses of electric power were estimated in the wires of traction network of feeder zones in the areas of one of Ukrainian railways electrified on a direct current $(+3.0 \mathrm{\kappa B})$. With this purpose we have got time dependences of the rectified feeder voltage and currents, performed their crosscorrelation-spectral treatment and determined electric indexes and losses of electric power $\Delta W_{\mathrm{b}}$ and $\Delta W_{\mathrm{ad}}$, daily dependences of which (in one of areas) are presented in fig. 2 but absolute and relative values in three areas are given in Table.

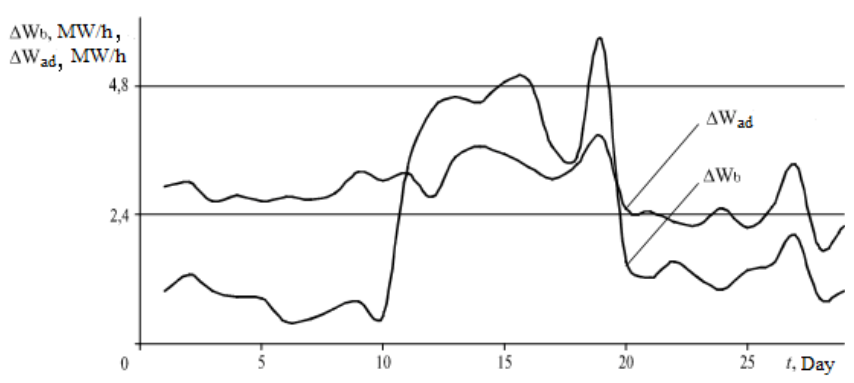

Fig. 1 - Daily average (for one month) values of basic $\Delta W_{\mathrm{b}}$ and additional $\Delta W_{\text {ad }}$ losses of electric power in a traction network of one of feeder areas

Fig. 2 and Table 1 shows that daily average values of non-productive losses of electric power, at first, exceed productive losses $\Delta W_{\mathrm{b}}$ by many days. Secondly, non-productive losses are 5.62...9.93\% (and maximal values - up to $15 \%$ ) of consumed (active) energy $W$ by electro-mobile composition and up to $\underline{140 \%}$ in comparison with productive losses. Consequently, non-productive (additional) losses exceed the productive losses of electric power almost by $40 \%$.

Then inactive power after Fryze will be written as 
Table 1

\begin{tabular}{|c|c|c|c|c|}
\hline \multirow[b]{2}{*}{$\begin{array}{l}\text { № } \\
\Pi / \Pi \\
\end{array}$} & \multirow[b]{2}{*}{ Types of electric power losses } & \multicolumn{3}{|c|}{ Daily average losses in areas } \\
\hline & & № 1 & № 2 & № 3 \\
\hline 1. & $\begin{array}{c}\text { Consumable (active) electric power, } W \text {, } \\
\mathrm{kW} / \mathrm{h}\end{array}$ & 28685 & 37872 & 24462 \\
\hline 2. & Productive (basic) losses, $\Delta W_{\mathrm{b}}, \mathrm{kW} / \mathrm{h}$ & 2085 & 2792 & 1357 \\
\hline 3. & Losses (additional) of loss, $\Delta W_{\text {ad }}, \mathrm{kW} / \mathrm{h}$ & 2850 & 3217 & 1362 \\
\hline 4. & Relation, $\Delta W_{\mathrm{ad}} / \Delta W, \%$ & 9.93 & 8.50 & 5.62 \\
\hline 5. & Relation, $\Delta W_{\mathrm{ad}} / \Delta W_{\mathrm{b}}, \%$ & 136.7 & 116.2 & 100.4 \\
\hline
\end{tabular}

\section{Conclusion}

1. General technological losses of electric power in traction networks are necessary to divide into productive and non-productive and estimate each of these components.

2. In the current conditions of limitedness of power resources, the most significant index of efficiency of traction power supply must be the level of non-productive losses of electric power.

\section{Literature}

[1] Kostin N., Reutskova O. Reactive power and NONProductive losses of electroenergy in electric transport of the direct current // Conference Proceedings "Modern Electric Traction" (MET 2009), Poland, Gdansk, -2009. -p.14-18

[2] Свешников А.A. Прикладные методы теории случайных функиий -М.: Наука, 1968. -463 c

[3] Венцель Е.С. Теория вероятности -М.: Наука, 1969. $-576 c$ 